Eutberz

$\mathfrak{i} \mathfrak{A} \mathfrak{a} \mathfrak{z} \mathfrak{w} \mathfrak{a} \mathfrak{b} \mathfrak{l}$

Zweiter $\mathfrak{B a n d}$ 



\title{
Eutber $\mathfrak{B}$ erte

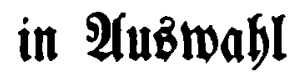

Unter Mitwirtung von 2lbert \{eikgmann

\author{
heraubgegeben \\ opt \\ Otto Clemen
}

Zweiter $\mathfrak{B a n d}$

Berlin
Berlag oon Bafter be Eruyter \& E.
1950 
Archiv-Nr. 321450

Druckgenehmigung: III 5794/49 - 7886/49

(4) F. Ullmun॥ G.m. b. H., Z wickau Sa., Talstr. 16-20 / Auftr. Nr. 493 